\title{
Duality Occurrences: Physical Origin of Wave Functions
}

\section{Louis-Marie Moukala}

Department of Exacts Sciences, École Normale Supérieure, Marien Ngouabi University, Brazzaville, Congo

\section{Email address:}

lmmouk@hotmail.fr

\section{To cite this article:}

Louis-Marie Moukala. Duality Occurrences: Physical Origin of Wave Functions. International Journal of Applied Mathematics and Theoretical Physics. Vol. 5, No. 1, 2019, pp. 15-19. doi: 10.11648/j.ijamtp.20190501.12

Received: January 23, 2019; Accepted: March 2, 2019; Published: March 21, 2019

\begin{abstract}
The quantum mystery began with the probabilistic interpretation of the wave function. However, this usefulness is definitive in Quantum Mechanics while the suspense continues. The present paper aims to investigate the origin of such a mystery. Hence, considering a relativistic charged particle in quantum vacuum, it appeared that: $(i)$ from a classical association, this electromagnetismself-consistent derives from the usual wave function, which corresponds to the scalar nature in addition to the vector one. (ii) Such duplicity is only justifiable when the related gauge fields describe fermions, in accordance with the previous theory of duality field-matter. This occurrence then corresponds to the appearance of bosons at cell intersections in vacuum lattice, whatever is the field. (iii) From the related gauge couplings, the scalar function must have an unknown vector companion. Both appear as originating the related conservation laws on one side. On the other side, specific variations of the field front would explain their physical origin. (iv) Moreover, both define an original gauge field to which that of law conservation is sensible. $(v)$ Due to definition validity in any reference system, their possible quantization should lead to that of scalar and vector fields of stationary states. At last, the results highlight the connection between waves and fields associable to any object, emphasizing the field unification framework.
\end{abstract}

Keywords: Cell Intersection, Duality Field-Matter, Gauge Coupling, Gauge Fermion, Gauge Field, Unified Field, Vacuum Lattice, Wave Function

\section{Introduction}

Quantum Mechanics and Relativity constitute the foundations of modern physics. However, if the latter is determinist, the former is not [1]. This began with the introduction ad hoc by Schrödinger of a wave function in its famous equation. This associates a wave to a particle in order to equationize de Broglie hypothesis. One interprets this function as the amplitude of probability density to find a particle at a given space position of a system. Its equation first interest was to justify the wave nature of particles, of course. Then this justified Bohr atomic model since the early times.The wave function isyet useful nowadays in many studies [2-3]; despite the field theories [4]. This function is relatable to the particle quantum state, which is a more general concept defined in Dirac formalism [5,6]. Although the predictions of the probabilistic interpretation are remarkably precise as never known in physics, one does not yet know the quantity originating that amplitude. Determining this should come to know the physical origin of the wave function. Beyond the measurement problem which relies on [7-9], that indetermination surely originates the socalled mystery in quantum physics. This appears in Bohr correspondence rule, where the classical equivalence of the wave function does not exist.

However, one could remedy such implication as it follows: "to an arbitrary quantity of a particle (alias the ghost), one associates a wave function". Such a quantity is understandable in classical physics when one associates a wave to an object. For instance, it seems evident that the hissing of a projectile in air is the perception of the wave produced by this object. This wave is justifiable by the displacement field of cells $[10,11]$. Therefrom, it is deductible that the wave function must have connection with such fields in vacuum. In quantum physics itself, one knows that electromagnetism structures material media at atomic scale; this is manifestable in different subfields defining the chemical links. Once again, one can assume that quantum vacuum also has such a field by analogy, manifestable in fundamental fields. This is certainly the spin or unified field previously emphasized [12]. Indeed, this differentiates into 
the four fundamental fields; each definable by a gauge coupling of fermions composing gauge bosons. For such reasons, wave functions must necessarily have origins, as it will appear here.

$\mathrm{n}$ the previous cited paper, it appeared that fermion quantum states are relatable to gauge fields for a given charge. Here, we would like to establish the connection between these and the so-known wave function. Hence, after establishing some useful foundations in the simplest relativistic case, we will analyze and discuss the physical occurrences.

\section{Foundations of Equivalence Wave-Field}

Consider a particle of mass $m$ and charge $q$, moving freely at $\vec{v}$ speed in vacuum. In the related model of vacuum lattice [12], this is similar to a material medium, which is homogeneous and isotropic. This section begins with a classical association, then screens the related happenings within the duality field-matter context.

\subsection{Classical Association of Field and Wave}

The relativistic classical association of the wave function to a particle relies on the relation of the relativistic energy $E^{2}=p^{2} c^{2}+m^{2} c^{4}$; where $\vec{p}$ is the momentum and $c$ is the speed of light. This yields Klein-Gordon equation with the traditional association of quantities to the related operators. The solutions of this are plane waves and seem not relatable to a specific field (see furtherFigure 1). However, any free particle has at least one self-consistent field. Logically, one has to associate this to the particle. Thus, if the 4-potential $|\mathrm{A}\rangle=(\overrightarrow{\mathrm{A}}, \mathrm{iV} / \mathrm{c})$ represents this for the charge $q$, one used to write the relation below [13], by substitutions of energy and momentum, with reference to electromagnetism classical definitions.

$$
(E-q V)^{2}=(\vec{p}-q \vec{A})^{2} c^{2}+m^{2} c^{4}
$$

However, that equation mixes both corpuscular and wavy natures. In practice, one needs to work with only one nature. This is possible only if one substitutes the dynamic quantities by the corresponding operators, as usual. However, such procedure is only complete when multiplying first that expression by an arbitrary function, on which will act the operators.

Let $\xi$ be this function. One can then apply Bohr correspondence rule with the substitutions $E \leftarrow \widehat{H}, p \longleftarrow \widehat{P}$ and $\xi \leftarrow \psi$; whereas $\widehat{H}$ is the Hamiltonian and $\widehat{P}$ is the impulse operator; $\psi$ is the wave function associated to the particle.One therefore obtains the equation.

$$
(\widehat{H}-q V)^{2} \psi=(\widehat{P}-q \vec{A})^{2} c^{2} \psi+m^{2} c^{4} \psi
$$

which constitutes the double association of a self-consistent field and the related wave to the particle. This exploitation depends on the operator definitions.

\subsection{The Ghost Identification}

When using ad hoc the classical definitions $\widehat{H}=i \hbar \partial / \partial t$ and $\widehat{P}=-i \hbar \vec{\nabla}$, their substitutions in the previous expression and this development let finding terms in $q$ and $q^{2}$. The corresponding expression is identical to that of Klein-Gordon (case of $q=0$ ), if the conditions below are satisfactory.

$$
\left\{\begin{array}{c}
V^{2}-c^{2} \vec{A}^{2}=0 \\
c^{2} \vec{\nabla} \vec{A}+\partial_{t} V=0 \quad ; \partial_{t}=\partial / \partial t \\
V \partial_{t} \psi+c^{2} \vec{A} \vec{\nabla} \psi=0
\end{array}\right.
$$

The second relation represents Lorenz gauge in electromagnetism. That is, $|\mathrm{A}\rangle$ corresponds to a gauge field having a transverse or vector nature. When comparing the third equation to the first one, one easily deducts the following proportionalities.

$$
\left\{\begin{array}{c}
\partial_{t} \psi=\alpha V \\
\vec{\nabla} \psi=-\alpha \vec{A}
\end{array} ; \alpha \in \mathbb{R}^{*}\right.
$$

That is, the gauge field derived from the wave function, which is then more fundamental. This can write

$$
\psi=\alpha \underbrace{\int V d t}_{\xi} \text { or } \psi=-\alpha \underbrace{\int \vec{A} d \vec{r}}_{-\xi}
$$

Leaving aside the constant, one can define the previous arbitrary function by putting $\xi=\int V d t$ or $\xi=-\int \vec{A} d \vec{r}$. Hence, theghost is the primitive in time of the scalar potential or that in space of the related vector potential. The result section examines its physical origin.

\subsection{Duplicity of Self-Consistent Field}

By taking the gradient of (4) first equation and the time derivative of the second, one obtains another gauge relation, i.e. $\vec{\nabla} V+\partial_{t} \vec{A}=\overrightarrow{0}$, which means that $\vec{A}$ is potential. That is, the gauge field $|\mathrm{A}\rangle$ has also a longitudinal or scalar nature, directly linked to the wave function. This is relatable to mass and is then gravitational. Such duplicity calls attention on the fact that theclassical definitions of electromagnetism, relative here to operator definitions, are rather incomplete. This comes in part from the sole consideration of the selfconsistent field of charge. That related to mass does not explicitly appear. The system (3) reduces to

$$
\left\{\begin{array}{c}
c^{2} \vec{\nabla} \vec{A}+\partial_{t} V=0 \\
\vec{\nabla} V+\partial_{t} \vec{A}=\overrightarrow{0}
\end{array}\right.
$$

As the first relation describes the conservation law of electric charge around the particle, the second one then describes that of mass (see below). One must have in fact two different gauge fields, which are proportional.

\subsection{Origin of the Proportionality}

In the duality field-matter, the existence of both scalar and vector gauge fields for any charge appears. These obey to the following gauges, when considering fundamental fermions, 
i.e. which travel at the speed of light $(v=c)$.

$$
\left\{\begin{array}{c}
c^{2} \vec{\nabla} \vec{A}_{v} \pm \partial_{t} V_{v}=0 \\
\vec{\nabla} V_{s} \pm \partial_{t} \vec{A}_{s}=\overrightarrow{0}
\end{array}\right.
$$

The sign + defines a gauge of fermions while the sign defines that of antifermions. One obtains four possible couplings, i.e. $(+,+),(-,-),(-,+)$ and $(+,-)$, where the first sign represents a scalar gauge field. Each couple corresponds to a fundamental field respectively identified as electromagnetic, gravitational, weak and strong. The first equations express the law of charge conservation in the related fields. The seconds express that of mass conservation, translatable by the equivalence principle, i.e. the fermion gravitation is equal to its acceleration. Of course, the scalar field is gravitational as indicated above, i.e. $\left[V_{s}\right]=m^{2} . s^{-2}$ and $\left[A_{s}\right]=m \cdot s^{-1}$. Each field must respect both gauges, at cell intersections (interfaces or tops) of the model of vacuum lattice.That is, both modes must be proportional. One can therefore deduct (wave) functions from solutions ofthe related field equations [14]. In any field, this corresponds to the appearance of virtual bosons, which assumethe wave propagation. In electromagnetismcase, these are virtual photons.

Figure 1 illustrates such bosons when quantum vacuum configures itself as a lattice of simplest cubic system; others are possible regarding a homogeneous and isotropic medium. In this, plane wave solutions represent the unified fieldstructuring quantum vacuum; such as both modes intersection corresponds to its differentiation. As for the solutions describing the field propagation, they must vanish with distance around the moving object (expressions (17) [12]).

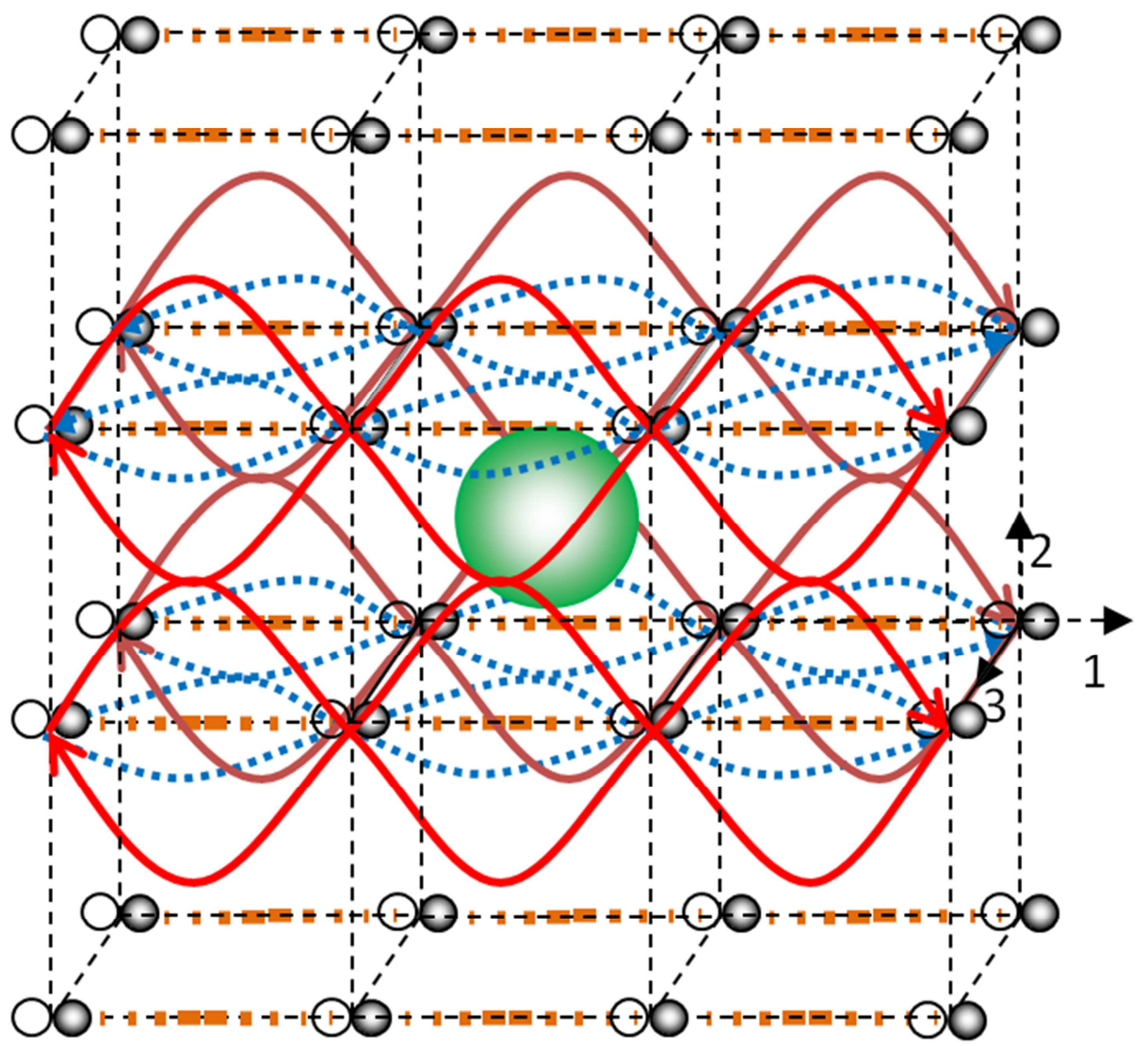

Figure 1. Virtual boson appearance in vacuum: the big sphere represents the moving object at a given position along the horizontal direction. The scalar gauge field $\vec{A}_{s}$ is parallel to this and delimits the cells (short-vertical lines). The axes origin reports to the object center. The sinusoids (solid or dot lines) illustrate, for one lattice layer, both possible transverse-plane solutions of $\vec{A}_{v}\left(\perp \vec{A}_{s}\right)$. One chose their amplitudes for coherence. The stationary solutions suit with the mono-crystalline lattice description. The couples at cube tops then correspond to virtual bosons $\left(A_{s}^{1}, A_{v}^{2}\right)$ or $\left(A_{s}^{1}, A_{v}^{3}\right)$ where meet both modes $($ null amplitudes). Boson amplitudes must however vanish according to the acting field.

\subsection{Vector Wave Function: A Coupling Imperative}

From (4), it appears that the variations of scalar wave function $\left(\psi=\psi_{s}\right)$ are due to the appearance of scalar gauge field, i.e. to mass existence. For any charge existence, one has to find another wave function. In electromagnetism and with respect to (7) first relation, Lorentz gauge allows putting

$$
\left\{\begin{array}{l}
V_{v}=\beta c^{2} \vec{\nabla} \vec{\psi}_{v} \\
\vec{A}_{v}=-\beta \partial_{t} \vec{\psi}_{v}
\end{array} ; \beta \in \mathbb{R}^{*}\right.
$$

Whereas $\vec{\psi}_{v}$ is the vector wave function for an electric charge. Therefrom, one can see that the variations of this are due to the vector gauge field $\left|A_{v}\right\rangle$, according to the charge conservation; $\vec{\psi}_{v}$ is then a quantity more fundamental like the 
scalar wave function.

As at cellular intersections of vacuum, both gauge fields are proportional (see above), one can write the relations

$$
V_{v}=\gamma V_{s} ; \vec{A}_{v}=\gamma \vec{A}_{s} ; \gamma \in \mathbb{R}^{*}
$$

From the previous equalities and (4) adaptation (index $(s)$ ), this yields the equations

$$
\left\{\begin{array}{c}
\alpha \beta c^{2} \vec{\nabla} \vec{\psi}_{v}=\gamma \partial_{t} \psi_{s} \\
\alpha \beta \partial_{t} \vec{\psi}_{v}=\gamma \vec{\nabla} \psi_{s}
\end{array}\right.
$$

One can distinguish three cases with respect to the constants. (i) When $\alpha \beta / \gamma=-1$, one obtains the same gauges of electromagnetism. However, as photon fermions are already fundamental, this is simply impossible. (ii) When $\alpha \beta / \gamma=1$, one obtains both gauges of gravitation satisfied by any 4 -vector proportional to $\left(\vec{\psi}_{v}, i \psi_{s} / c\right)$ - see (7) for the signs. That is, electromagnetism derives from gravitation or the former can originate the variations of the latter. This implies the known sensibility of photons to gravitation in General Relativity. (iii) The last case $\alpha \beta / \gamma \neq$ \pm 1 does not match with any gauge of fundamental field. This should correspond to the undifferentiated unified field.

This approach is applicable to other couplings from (7) when deducting both scalar and vector functions from proper gauges. The first condition always corresponds to the initial field itself and the second to the opposite gauge coupling. With respect to the sensibilities, one can thus verify that strong field should generate the variations of weak field. This opposite gauge coupling is strong or relatable to its anti-field. Hence, it suits assuming that the weak field generates the variations of unified field;this can furthermore differentiate into electromagnetism or gravitation according to the charge of implied bosons. Such discontinuity matches with the fact that not all the weak bosons have electric charge, e.g. the boson $Z^{0}$, [15].

Those relations of cause to effect define the inclusive hierarchy order illustrated in Figure 2. From this, one hasto assume that gravitation could only generate the variations of unified field (see third condition above).

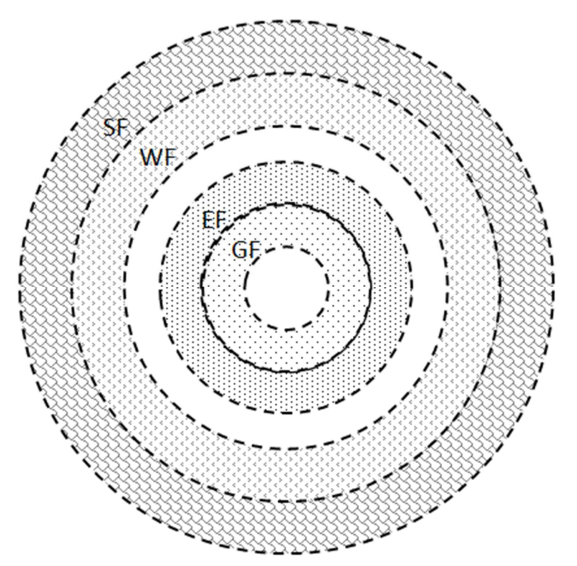

Figure 2. Fundamental field hierarchic order in virtual bosons: $S F=$ Strong Field; $W F=$ Weak Field; EF = Electromagnetic Field; GF = Gravitational Field. The blank areas represent the unified field. Each outer field generates the variations of the next.

\section{Results and Discussion}

These emphasize the existence of both kinds of wave functions and these physical origins; their validity in any reference system as well.

\subsection{Main Occurrences of the Duality}

The existence of both kinds of gauges from one electromagnetic-like gauge field appeared evident (see relations (6)). This means that both vector and scalar fields must always exist together. Hence, Maxwell equations only describe the former. The related theory should then regard fermions instead of bosons, contrarily to what one used to admit. This is striking asalready noted in the reference [10]. A photon composition is not yet proved, certainly because its gauge fermions should be confined. However, one can ask "what quantum particles describe the static electric and magnetic fields".

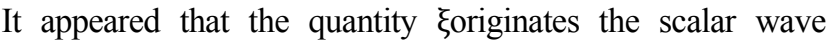
function (see (5)) andthe definition remains valid at any position (see further). Owing to the gravitational nature of any scalar field (see subsection 2.4), $\xi$ is homogeneous to an areolar speed $\left([\xi]=m^{2} \cdot s^{-1}\right)$, whichsuggests the variation of a surface in course of time. When considering spherical coordinates from the object origin, such a surface is assimilableto that of the field front around the object, at a given distance. Figure 3 tries to show the quantizable distribution of virtual bosons at cell intersections. The propagation of the decreasing field sweeps a surface. Hence, it suits to deduct that the physical quantity originating the wave function is the expansion speed of the fieldfront surface.

It also appeared that the gauge couplings imply the existence of scalar and vector wave functions. It seems that the latter is unknownin literature. From the second relation of (8), the associable quantity is the integral part of $\vec{\psi}_{v}=-(1 / \beta) \int \vec{A}_{v} d t$. As at cell intersections both vector potentials are proportional $\left(\vec{A}_{v} \propto \vec{A}_{s}\right)$, one also obtains $\vec{\psi}_{v} \propto$ $\int \vec{A}_{s} d t$. This integral representsa displacement. Hence, one can deduct that the field front displacement originate the vector function.

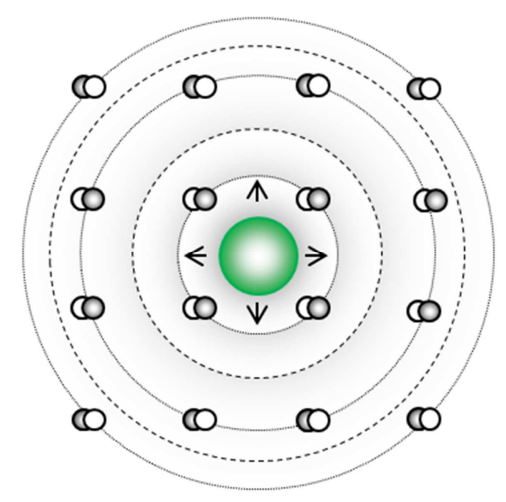

Figure 3. Distribution of virtual bosons around the test object: the circle set belongs to one of the three planes such as the boson number is limited on the related sphere, e.g. there is height on the first (see Figure 1). Each boson appears at cell intersection where the unified field differentiates. The arrows indicate the field-front expansion. The dashed lines indicate this at intermediary positions between cells. 
Moreover, both wave functions allow connecting one field to another such as the initial should generate the variations of its primitive at cell intersections of vacuum lattice (see Figure 2). This also brought out the fact that the weak field is particular. Some weak bosons have zero electric charge contrarily to other kinds, which should have charges of the next field of the hierarchy order. Inside cells however, the relations between fields are undeterminable, due to gauge field independency. These findings are relevant.

\subsection{Theory Validity in any Reference System}

All of the previous results regard the case of inertial systems, as indicated before. That of non-inertial systems is deductible when the gauge fields do no longer represent selfconsistent fields. Given that any of these also represents a fundamental field, one must always have the same gauges. Hence, the relations do not change between gauge fields and wave functions (see subsection 2.5). They are therefore valid for non-inertial systems too, which imply stationary virtual bosons.

Thus, in case of quantization of the usual wave function, one can deduct that of the related scalar field (see (4)); consequently, that of the related vector field at cell intersections (see (9)). For instance, the quantized wave function of hydrogen atom should allow deducting the quantization of the electromagnetic field of the system from that of its inherent gravitational field (see above). This is germane.

\section{Conclusion}

The double association, to a relativistic free particle, is examinedbetween the usual wave function and its electromagnetic self-consistent field. It appeared the following. ( $i$ ) This function derivatives are proportional to the components of the self-consistent gauge field, which is scalar and vectorial; both natures appear inseparable for an electromagnetic field description. (ii) Such duplicity is justifiable from the duality field-matter, as intersection conditions between cells in the model of vacuum lattice.

As well (iii) the physical quantity relatable to that scalar function isthe circulation of vector potential; the dynamic meaning is discussed. (iv) This function has a vectorial homologous owing to the field charge. (v) This allowed showing the possible appearance of field sensibilities with respect to others. For instance, the description of the wave nature of photons, with both functions, is attributable to photon gravitation.

Finally, the existence of two kinds of (wave) functions in any kind of system appears. The set of both should be relatable to Dirac's wave function of four components. Their preexistence, which is attributable to vacuum structuring, emphasizes precedence between fields one can investigate consequences; the gauge boson motion as well.

\section{References}

[1] C. Garola. and J. Pykacz. (2004). Locality and measurement within the SR model for an objective interpretation of quantum mechanics. Found. Phys. 34, 449-475.

[2] S. Zeller (2018). Determination of the He-He, Ne-Ne, Ar-Ar, and $\mathrm{H} 2$ interaction potential by wave function imaging. Phys. Rev. Lett. 121, 083002.

[3] Wataru Kohno, Akimitsu Kirikoshi, Takafumi Kita (2018). Ground-State Wave Function with Interactions between Different Species in $M$-Component Miscible Bose-Einstein Condensates. Journal of the Physical Society of Japan 87, 034002 .

[4] I. V. Kanatchikov (2018). The solution of Schrodinger equation justifies early Bohr atomic model. Rep. Math. Phys. $82,373$.

[5] P. A. M. Dirac. The Principles of Quantum Mechanics. Oxford: Clarendon Press, 1958.

[6] L. E. Ballentine. Quantum Mechanics: A modern development. Singapore: World Scientific Publishing Co, 1998, pp. 234-240.

[7] E. Tammaro (2014). Why Current Interpretations of Quantum Mechanics are Deficient. arXiv: quant-ph/1408.2093v2

[8] P. L. Saldanha (2018). Inconsistency of a realistic interpretation of quantum measurements: A simple example. arXiv: quant-ph/1805.05446v3

[9] M. Schlosshauer (2004). Decoherence, the measurement problem and interpretations of quantum mechanics. Physics Faculty Publications and Presentations 13. http://pilotscholars.up.edu/phy_facpubs/13

[10] L. M. Moukala (2018). Characterization of cubic crystalline systems: a field theory uniting elasticity with electromagnetism. Res. J. Material Sci. 6, 1-4.

[11] J. L. Davis. Wave propagation in solids and fluids. New York: Springer-Verlag, 1988, pp. 274-299.

[12] L. M. Moukala (2018). Ultimate duality field-matter: field structural unification. Res. J. Recent Sci. 7, 1-6.

[13] A. Messiah. Mécanique quantique. Dunod: Paris, 1959, pp. 56-57.

[14] L. M. Moukala (2017). The unified field as vacuum quintessence in waves equations. Res. J. Physical Sci. 5, 1-6.

[15] C. Patrignani et al. (2016 and 2017 update). Particle Data Group. Chin. Phys. C 40 100001. http://pdg.lbl.gov 